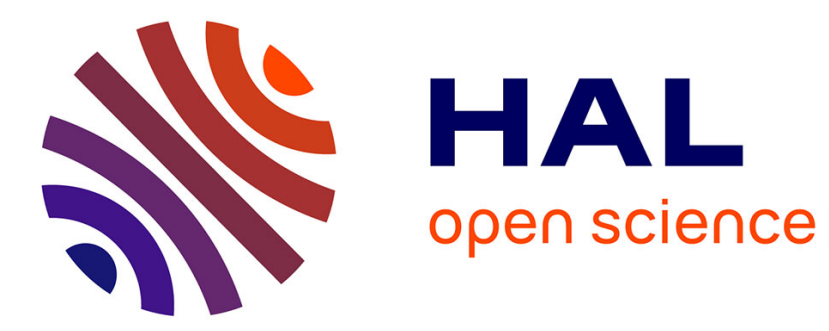

\title{
Inertial collapse of liquid rings
}

Baptiste Darbois Texier, Keyvan Piroird, David Quéré, Christophe Clanet

\section{To cite this version:}

Baptiste Darbois Texier, Keyvan Piroird, David Quéré, Christophe Clanet. Inertial collapse of liquid rings. Journal of Fluid Mechanics, 2013, 717, pp.R3. 10.1017/jfm.2013.15 . hal-00996421

\section{HAL Id: hal-00996421 \\ https://hal-polytechnique.archives-ouvertes.fr/hal-00996421}

Submitted on 27 May 2014

HAL is a multi-disciplinary open access archive for the deposit and dissemination of scientific research documents, whether they are published or not. The documents may come from teaching and research institutions in France or abroad, or from public or private research centers.
L'archive ouverte pluridisciplinaire HAL, est destinée au dépôt et à la diffusion de documents scientifiques de niveau recherche, publiés ou non, émanant des établissements d'enseignement et de recherche français ou étrangers, des laboratoires publics ou privés. 


\title{
JFM RAPIDS journals.cambridge.org/rapids
}

\section{Inertial collapse of liquid rings}

\author{
Baptiste Darbois Texier ${ }^{1,2}$, Keyvan Piroird ${ }^{1,2,} \dagger$, David Quéré ${ }^{1,2}$ \\ and Christophe Clanet ${ }^{1,2}$
}

\author{
${ }^{1}$ PMMH, UMR 7636 CNRS, ESPCI, Université Paris 6, Université Paris 7, 75005 Paris, France \\ ${ }^{2}$ Ladhyx, UMR 7646 CNRS, École Polytechnique, 91128 Palaiseau, France
}

(Received 3 October 2012; revised 18 December 2012; accepted 2 January 2013;

first published online 7 February 2013)

Liquid rings can be generated in the Leidenfrost state using liquid oxygen of low boiling point $\left(-183^{\circ} \mathrm{C}\right)$ and high magnetic susceptibility, allowing one to 'sculpt' the liquid into a ring shape using an annular magnet. When the magnetic field is turned off, the ring shrinks back into a puddle with a constant acceleration. A potential flow approach accurately describes the dynamics of closure with an equation reminiscent of the Rayleigh-Plesset equation for the collapse of transient cavities.

Key words: drops and bubbles, interfacial flows (free surface), pinch-off

\section{Introduction}

It is commonly said that liquids have no specific shape and adopt that of their container. This is true under gravity as long as surface tension can be neglected. Otherwise, these forces tend to minimize the surface area of a given volume, leading to rounded and smooth shapes - spheres in the absence of other forces. Toroidal or ring-shaped drops are therefore rather scarce and often result from dynamical situations. Joseph Plateau in his pioneering work observed such shapes by imposing a rotation on a droplet suspended in a bath of another liquid (Plateau 1863; Brown \& Scriven 1980). Worthington (1879) studied the spontaneous segmentation of a liquid annulus made of mercury, formed with a mould that is rapidly removed. Centrifugal force generated by the rolling motion of viscous non-wetting drops on an inclined plane can lead to a toroidal shape (Aussillous \& Quéré 2004), also obtained during an impact on a solid surface (Bartolo, Josserand \& Bonn 2006) or with a liquid drop falling in another liquid (Kojima, Hinch \& Acrivos 1984; Sostarecz \& Belmonte 2003). Recent experimental (Pairam \& Fernandez-Nieves 2009) and theoretical (Yao \& Bowick 2011) studies have focused on the dynamics and/or instability of toroidal droplets in the limit of low Reynolds number. Pairam \& Fernandez-Nieves (2009) observed that a toroidal drop formed by extrusion of a liquid into a viscous bath either

$†$ Email address for correspondence: keyvan.piroird@doc.polytechnique.org 

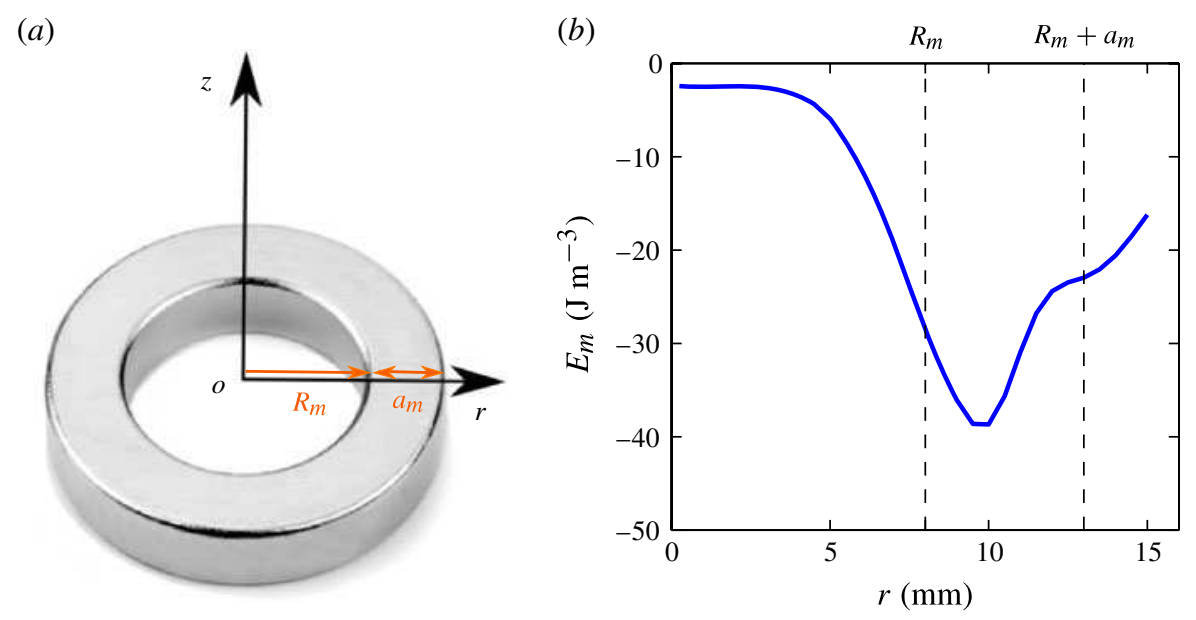

FigURE 1. (a) Annular neodymium magnet of inner radius $R_{m}=8 \mathrm{~mm}$, width $a_{m}=5 \mathrm{~mm}$ and height of $5 \mathrm{~mm}$. (b) Magnetic energy per unit volume $E_{m}$ as a function of radial coordinate $r$ for this magnet, at a given distance $z=2 \mathrm{~mm}$ above it. Dashed lines represent the position of the magnet.

breaks into droplets, or shrinks, depending on its aspect ratio. In the latter case, the shrinking velocity is constant and depends on the aspect ratio. This problem has also been studied experimentally (McGraw et al. 2010) and numerically (Nguyen et al. 2012) at a nanometre scale.

We show in the present work that it is possible to create a liquid ring in the Leidenfrost state, using liquid oxygen. Because of its low boiling point $(90 \mathrm{~K})$, this cryogenic liquid placed on a solid at room temperature levitates on a cushion of its own vapour, which strongly reduces friction and acts as a thermal insulator. Moreover, liquid oxygen is paramagnetic and can thus be manipulated by magnetic fields (Piroird, Clanet \& Quéré 2012a). In a sufficiently intense field, liquid oxygen can be trapped and sculpted into various shapes, by varying the design of the magnet approaching it. Above an annular magnet, oxygen rings can be formed and stabilized, and we study here how the ring relaxes when the field is suddenly switched off.

\section{Rings of liquid oxygen}

In order to create a liquid ring, an annular neodymium magnet of inner radius $R_{m}$ and width $a_{m}$ (figure $1 a$ ) is placed under a horizontal glass plate of thickness $2 \mathrm{~mm}$. Liquid oxygen poured on the glass plate spontaneously adopts the shape of a ring under the influence of the magnetic field, as shown in figure $2(a)$ and sketched in figure $2(b)$. Using different magnets, we can vary the aspect ratio of the ring. The inner radius $R_{o}$ of the liquid annulus has been varied between 2 and $10 \mathrm{~mm}$ and the width $a_{o}$ between 1.5 and $4.5 \mathrm{~mm}$, so that the aspect ratio, defined as $\pi\left(2 R_{o}+a_{o}\right) / a_{o}$ varies from 6 to 21. The magnetic energy per unit volume of liquid oxygen is:

$$
E_{m}=-\frac{\chi}{2 \mu_{o}} \boldsymbol{B}^{2}
$$


(a)

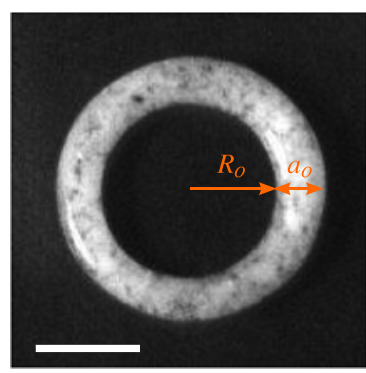

(b)

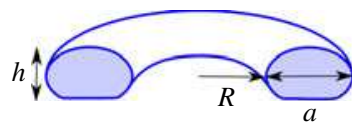

(c)

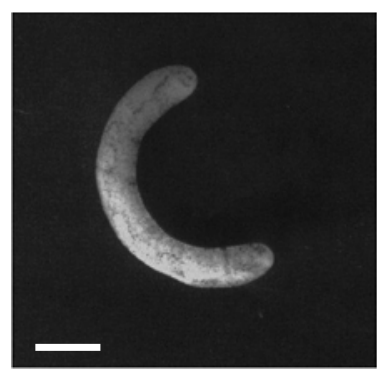

FIGURE 2. (a) Top view of a liquid ring of oxygen sitting on glass in the Leidenfrost state. The ring is obtained by placing an annular magnet $\left(R_{m}=4.5 \mathrm{~mm}, a_{m}=1.5 \mathrm{~mm}\right)$ below a $2 \mathrm{~mm}$-thick glass plate. Here, $R_{o}=4 \mathrm{~mm}$ and $a_{o}=2.2 \mathrm{~mm}$ (aspect ratio $\left.\pi\left(2 R_{o}+a_{o}\right) / a_{o}=14.5\right)$. The white spots are small ice crystals. The bar represents $5 \mathrm{~mm}$. (b) Sketch of side view of the liquid ring, and definition of its characteristic lengths. (c) C-shaped puddle $\left(R_{o}=6.25 \mathrm{~mm}, a_{o}=2.8 \mathrm{~mm}\right)$ obtained above an annular magnet $\left(R_{m}=4.75 \mathrm{~mm}, a_{m}=4.75 \mathrm{~mm}\right)$ when the volume of liquid oxygen is too small to form a complete ring. The bar represents $5 \mathrm{~mm}$.

where $\chi$ is the magnetic susceptibility $(0.0035$ in SI units at $90 \mathrm{~K}), \mu_{o}$ the magnetic permeability of vacuum $\left(4 \pi \times 10^{-7} \mathrm{H} \mathrm{m}^{-1}\right)$ and $\boldsymbol{B}$ the magnetic field, close to $0.5 \mathrm{~T}$ on the magnet's surface. With a Hall effect teslameter, the magnetic field can be measured as a function of the radial coordinate $r$ in the plane of the glass plate, which gives the map of the magnetic energy shown in figure $1(b)$. The slope at a given point is the radial force per unit volume exerted by the magnet on the liquid (a negative slope corresponds to a positive radial force). The central position $(r=0)$ is an unstable equilibrium position: the liquid sits in a region around $r=R_{m}+a_{m} / 2$, which minimizes the magnetic energy. The liquid is therefore radially confined around this point and forced to extend in the orthoradial direction. If the volume of liquid oxygen is large enough, we eventually obtain a liquid ring (figure $2 a$ ). If the volume is too small, there is not enough liquid to fill the magnetic well. The ring cannot be closed and we obtain a $\mathrm{C}$-shaped puddle (figure $2 c$ ). The width of this particular shape is fixed by a balance between gravity and magnetic confinement; adding more oxygen increases the angular extent of the liquid and there is a continuous transition to a ring of the same width. On the other hand, if a ring is left to evaporate, it becomes more and more narrow until it breaks and becomes a C-shape when the width $a_{o}$ is of the order of the height $h$. The ' $\mathrm{C}$ ' then opens until its width matches again the width $a_{m}$ of the magnet.

These liquid rings are stabilized by the magnetic field (when wide enough) but subjected to a complex internal flow revealed by the motion of the small white crystals present in the oxygen (ice, resulting from the condensation of water vapour). The inner radius $R_{o}$ and width $a_{o}$ of the liquid ring are comparable to that of the magnet. The volume of liquid $\Omega$ involved is always large compared to the cube of the capillary length of liquid oxygen $l_{c}=\sqrt{\gamma / \rho g}=1.1 \mathrm{~mm}$, where $\gamma=13 \mathrm{mN} \mathrm{m}^{-1}$ (Bembenek 2006) and $\rho=1140 \mathrm{~kg} \mathrm{~m}^{-3}$ (Lide 2010) are the liquid surface tension and density at $90 \mathrm{~K}$, and $g$ the gravitational acceleration. The rings that we create are puddles with $a_{o}>l_{c}$. The cross-section is oblong rather than circular, as sketched in figure $2(b)$. This is why we call them 'rings' rather than 'tori'. In the absence of magnetic field, the height $h$ (figure $2 b$ ) is given by a balance between gravity and capillary forces 

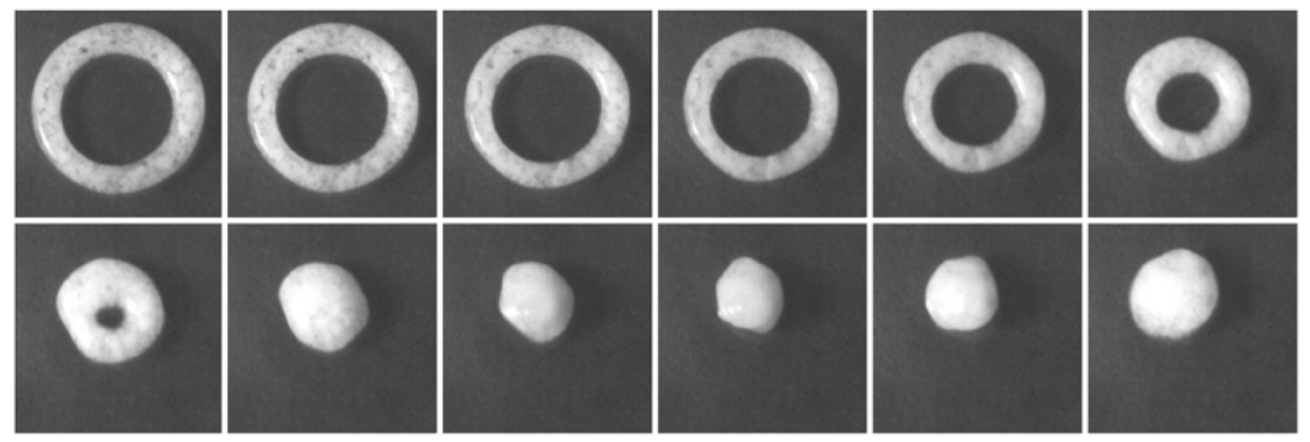

FIGURE 3. Top view of a collapsing liquid oxygen ring, of initial inner radius $R_{o}=4 \mathrm{~mm}$ and initial width $a_{o}=2 \mathrm{~mm}$. Time interval between pictures: $8 \mathrm{~ms}$. The corresponding movie is available as a supplemental material at http://dx.doi.org/10.1017/jfm.2013.15.

and is equal to $2 l_{c}=2.2 \mathrm{~mm}$, whatever the volume of the ring, in the limit where $\Omega>l_{c}^{3}$. In the presence of a vertical gradient of magnetic field, the ring is more flat because the additional vertical magnetic force acts as an enhanced gravity: $h$ scales as a modified capillary length which decreases as the field increases (Piroird et al. 2012b). In our experiments, this effect is negligible for the small magnets and it can reach approximately $10 \%$ for the large ones.

Once the ring is formed, the magnet is rapidly removed and we observe from the top with a high-speed camera how the shape evolves in time (figure 3). The annulus shrinks in less than $0.1 \mathrm{~s}$, and the liquid is driven back to a 'pancake' shape for which surface tension is balanced by gravity. After the collapse, the kinetic energy acquired during the closure is converted into vibrations of the puddle (the last images in figure 3) that are slowly damped because of the weak friction. Figure 4 shows quantitatively the time evolution of the hole radius $R$ for different geometries, where $R$ is measured from the surface area of the hole in the ring, from which we deduce the equivalent radius of a circle of the same surface area. The closure velocity $\dot{R}$ is zero at the onset and it increases as a function of time. The radius seems to follow a parabolic law $R(t)=R_{o}-\Gamma t^{2} / 2$ (solid lines in figure 4), where $\Gamma$ is a constant acceleration of closure, discussed in the next section. For large ring magnets (symbol $x$ in figure 4) with stronger magnetic energy, removal of the magnet induces a quick increase in the ring height within the first $10 \mathrm{~ms}$ of the motion, which can result in small oscillations of the inner radius about the parabolic line. Even in the range of large aspect ratio explored (from 6 to 21), the ring never breaks into droplets, contrasting with the observations by Pairam \& Fernandez-Nieves (2009) in the viscous regime, where break-up systematically occurs for tori with aspect ratios larger than 6 .

\section{Model}

The absence of contact between the liquid and its substrate dramatically reduces friction. Consequently, the Reynolds number $R e=\rho U a / \eta$ based on the smallest length $a$ in the system is high: for a typical velocity $U \sim 0.1 \mathrm{~m} \mathrm{~s}^{-1}$ and a dynamical viscosity $\eta \sim 0.2 \mathrm{mPa}$ s (Lide 2010), we obtain $R e \sim 1000$. Assuming that the ring is at rest at the beginning of the experiment, Kelvin's circulation theorem ensures that the vorticity remains constant and equal to zero as the ring shrinks. We can therefore use a 


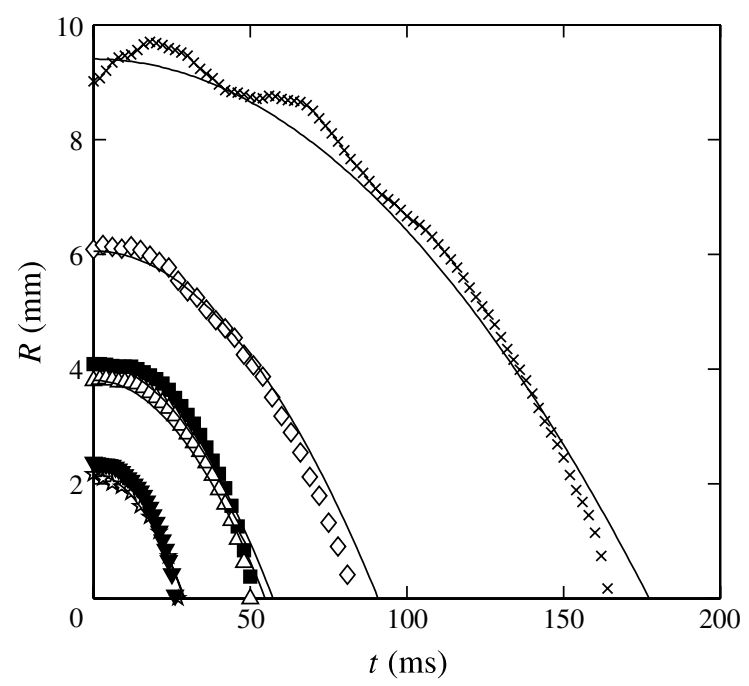

FIgURE 4. Radius $R$ of the liquid ring as a function of time for different initial aspect ratios. $\times$, $R_{o}=9.7 \mathrm{~mm}, a_{o}=3.1 \mathrm{~mm} ; \diamond, R_{o}=6.1 \mathrm{~mm}, a_{o}=2.9 \mathrm{~mm} ; \boldsymbol{\square}, R_{o}=4.0 \mathrm{~mm}, a_{o}=2.2 \mathrm{~mm} ; \Delta$, $R_{o}=3.8 \mathrm{~mm}, a_{o}=2.8 \mathrm{~mm} ; \boldsymbol{\nabla}, R_{o}=2.4 \mathrm{~mm}, a_{o}=1.6 \mathrm{~mm} ; \star, R_{o}=2.2 \mathrm{~mm}, a_{o}=2.0 \mathrm{~mm}$. Solid lines represent parabolic behaviour of the equation $R=R_{o}-\Gamma t^{2} / 2$, where $\Gamma$ will be shown in figure $5(a)$.

potential flow approach and describe the dynamics with the Bernoulli equation:

$$
\left[\rho \frac{\partial \phi}{\partial t}+\rho \frac{\boldsymbol{u}^{2}}{2}+p\right]_{B}^{A}=0
$$

where $\phi$ is the velocity potential, $\boldsymbol{u}=\nabla \phi$ the velocity field, $p$ the pressure field and $A$ and $B$ two points in the fluid. Since our problem has a radial symmetry, $\phi, \boldsymbol{u}$ and $p$ only depend on the radial coordinate $r$ and time $t$. We choose point $A$ at the outer radius of the ring $(r=R+a)$ and $B$ at the inner radius $(r=R)$ in order to obtain a differential equation for $R$.

Assuming a radial flow and a constant ring height equal to $h=2 l_{c}$, the mass flux through any annular section of radius $r$ between $R$ and $R+a$ is $\rho 2 \pi r u_{r}(r) h$. It has to be equal to the flux at $r=R$, that is, $\rho 2 \pi R \dot{R} h$ (the dot stands for time derivative). This conservation of mass leads to the following equation for the velocity field:

$$
\boldsymbol{u}(r)=\frac{R \dot{R}}{r} \boldsymbol{e}_{r}
$$

from which we deduce the potential $\phi(r)=R \dot{R} \ln \left(r / R_{\infty}\right)$, where $R_{\infty}$ is a constant. Finally, the pressure is given by the Laplace law: we have $p(R) \approx p_{o}+\gamma(2 / h-1 / R)$ and $p(R+a) \approx p_{o}+\gamma(2 / h+1 /(R+a))$. All this together leads to the following evolution equation for $R$ :

$$
\frac{\mathrm{d}(R \dot{R})}{\mathrm{d} t} \ln \left(\frac{R+a}{R}\right)+\frac{\dot{R}^{2}}{2}\left(\frac{R^{2}}{(R+a)^{2}}-1\right)=-\frac{\gamma}{\rho R}\left(1+\frac{R}{R+a}\right) .
$$




\section{B. Darbois Texier, K. Piroird, D. Quéré and C. Clanet}

This equation is reminiscent of the Rayleigh-Plesset equation governing the collapse of a cavitation bubble (Plesset \& Prosperetti 1977; Hilgenfeldt et al. 1998). It presents an even closer analogy with the collapse of a cylindrical transient cavity created, for example, by the impact of a solid body in a liquid at high Reynolds numbers (Duclaux et al. 2007; Bergmann et al. 2009). In such a case, points $A$ and $B$ used in (3.1) are respectively chosen behind the cavity and far from the hole, where liquid is at rest (both points are at the same altitude). The velocity potential is the same and the pressure is set by the hydrostatic pressure. Hence the differential equation for the radius of the cavity at a given altitude $z$ is:

$$
\frac{\mathrm{d}(R \dot{R})}{\mathrm{d} t} \ln \left(\frac{R}{R_{\infty}}\right)+\frac{\dot{R}^{2}}{2}=g z .
$$

Contrasting with our case, the constant $R_{\infty}$ does not disappear and plays the role of a cut-off length which has to be evaluated in order to estimate the order of magnitude of the logarithmic term (Gekle et al. 2009). In our case, the cut-off is simply the width of the ring. In addition, we have $R \gg a$ during most of the closure, so that we can linearize (3.3). Moreover, since the thickness $h$ of the ring is constant, the product $R a$ also remains constant by conservation of volume. Denoting $R_{o}$ and $a_{o}$ as the initial radius and width of the liquid ring, we eventually obtain:

$$
\ddot{R}=-\frac{2 \gamma}{\rho R_{o} a_{o}} .
$$

This equation predicts a constant acceleration $\Gamma$ of the inner radius $R$, where $\Gamma$ depends on the initial geometry and on the fluid surface tension and density. Integrating (3.5) twice using the conditions $\dot{R}=0$ and $R=R_{o}$ at $t=0$ provides the dynamics of closure for $R \gg a$ :

$$
R(t)=R_{o}-\frac{\gamma}{\rho R_{o} a_{o}} t^{2} .
$$

This parabolic behaviour is in good agreement with the observations in figure 4, whatever the aspect ratio of the ring. Values of the acceleration $\Gamma$ can be deduced from the fits with the data in figure 4, and compared to the value expected from (3.5). A good agreement is found in figure 5(a) between the model and the data, without any adjustable parameter. In addition, we can deduce from (3.6) the closing time $\tau=\sqrt{\rho R_{o}^{2} a_{o} / \gamma}$, considering that this equation describes the motion from the beginning to the end of the closure. This time is found to agree well with measurements, although always slightly overestimating them (by approximately $10 \%$ ), as shown in figure 5(b). Indeed, we can note in figure 4 that, close to the end, the collapse dynamics is quicker than the parabolic behaviour of (3.6), as discussed in the next section.

\section{Discussion}

These experiments can be completed by a few remarks.

(a) At the very end of the collapse $(R<a / 2)$, the radius $R$ tends to zero while $a$ tends to a constant defined by conservation of volume. In this limit, the hole radius follows a different behaviour, which is found in figure 6 to be a scaling law: $R$ scales as $(\tau-t)^{2 / 3}$, where $\tau$ is the instant at which the hole disappears $(R(\tau)=0)$. This scaling is characteristic of situations where capillarity opposes inertia: balancing the dynamic pressure $\rho(R /(\tau-t))^{2}$ with capillary pressure $\gamma / R$ leads 

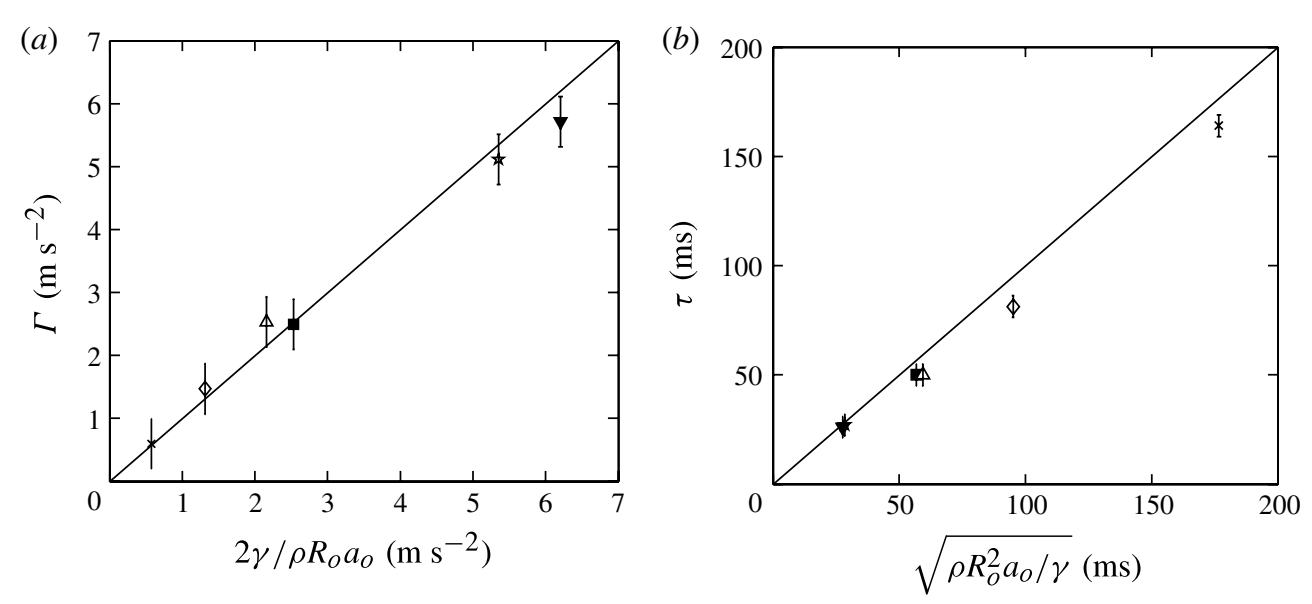

FIgURE 5. (a) Measured acceleration $\Gamma$ as a function of the theoretical prediction given by (3.5). (b) Measured closing time $\tau$ as a function of $\sqrt{\rho R_{o}^{2} a_{o} / \gamma}$, predicted by the model. The symbols are as in figure 4 and the solid line is $y=x$.

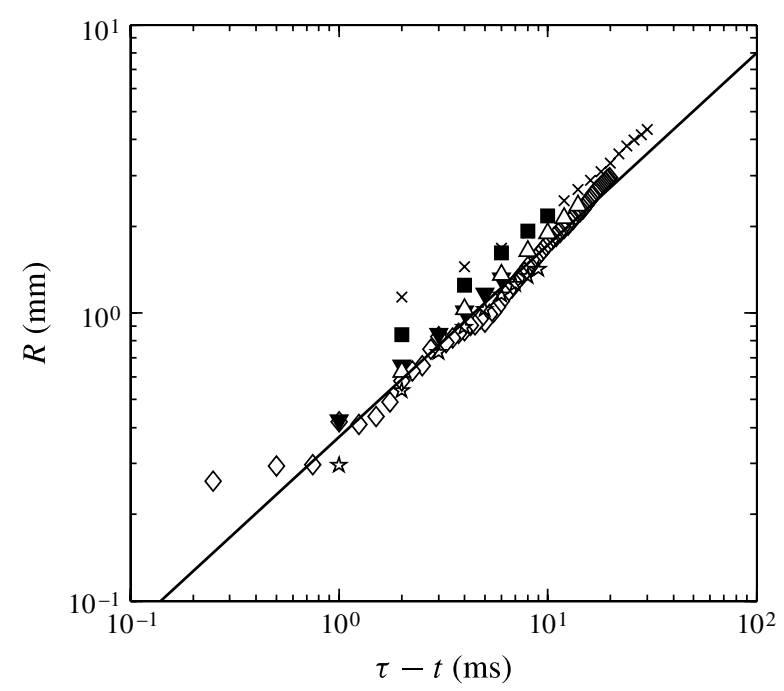

FiguRE 6. Hole radius $R$ at the very end of collapse $(R<a / 2)$ as a function of $\tau-t$, where $\tau$ denotes the closure time. Symbols are as in figure 4 . The solid line represents $R \propto(\tau-t)^{2 / 3}$.

to $R \sim\left(\gamma(\tau-t)^{2} / \rho\right)^{1 / 3}$, represented by a solid line in figure 6 with a prefactor of 1.65. The same behaviour is observed during the capillary pinch-off of an inviscid fluid (Chen \& Steen 1997; Day, Hinch \& Lister 1998; Leppinen \& Lister 2003). Yet, the asymptotic behaviour of (3.3) in the limit $R \ll a$ is found, both numerically and analytically, to be $R \sim(\tau-t)^{1 / 2}$. The model may not be suitable in this limit since it does not consider the ring meniscus, that might give rise to vertical pressure gradient 

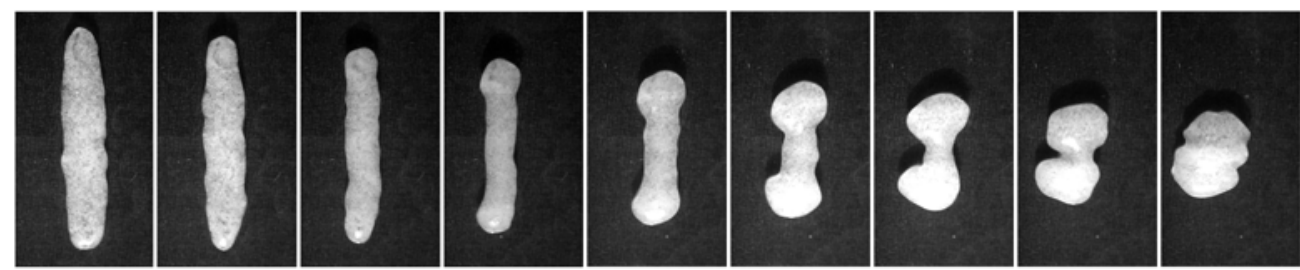

FIGURE 7. Top view of the retraction of a liquid ribbon of initial length $22.5 \mathrm{~mm}$, width $4 \mathrm{~mm}$ and height $2.2 \mathrm{~mm}$. The ribbon retracts from its two ends, without developing a Plateau-Rayleigh instability. Time interval between images: $12 \mathrm{~ms}$.

close to collapse. We only considered pressure differences between the ring inner and outer radii and we did not take into account that the inner radius $R$ depends on the altitude $z$ : it is minimal at roughly $z=h / 2$ and maximal at $z=0$ and $z=h$, giving rise to a vertical variation of the Laplace pressure $-\gamma / R(z, t)$. Just before the collapse, the pressure at the inner radius diverges at $z=h / 2$ while it is still of order $-\gamma / a$ at $z=0$ and $z=h$. Hence, the assumption of a simple two-dimensional flow does not hold at the end of closure and a more detailed description of the flow is needed to quantitatively explain the data.

(b) Our liquid rings never break up into several pieces, as could be expected from a Plateau-Rayleigh mechanism. We interpret the absence of capillary instability from the fact that the ring cross-sections are oblong rather than circular. These objects are closer to liquid sheets (that are intrinsically stable (Eggers 2011)) than liquid filaments, so that they can be stabilized by gravity. The ratio $a / h$ is initially equal to or larger than unity, and it increases during the movement. In this limit, a perturbation of the shape does not lead to a change in the height $h$ fixed by the balance between gravity and capillarity. If the radius of curvature $2 / h$ of the cross-section remains constant, any peristaltic variation of the shape only affects $a$ and necessarily increases the surface area. The Plateau-Rayleigh instability is only expected if $a<l_{c}$, a condition not satisfied for the rings, since it would lead to the formation of $\mathrm{C}$-shapes. This highlights the fact that if the ring is initially stable, it will remain stable during the collapse.

This scenario is confirmed by considering other shapes such as liquid ribbons, obtained by placing liquid oxygen above a parallelepipedic magnet. Figure 7 shows such a ribbon, of length $l=22.5 \mathrm{~mm}$, width $a=4 \mathrm{~mm}$ (aspect ratio $l / a=5.6$ ) and height $h=2.2 \mathrm{~mm}$ retracting after switching off the field. The ribbon goes through a dumbbell shape before collapsing back into a puddle. For this 'open ring', the retraction is driven by the ends, where the curvature of the surface is maximal. The length of the ribbon decreases linearly in time, with a velocity of $18 \mathrm{~cm} \mathrm{~s}^{-1}$. The C-shaped puddle (figure $2 c$ ) obtained by a partial filling of the annular magnetic well is another case of an open ring, which similarly to the straight ribbons, retracts from both ends without developing a Plateau-Rayleigh instability.

\section{Conclusion}

We have showed that the dynamics of closure of a Leidenfrost liquid ring results from a balance between inertia and capillarity, leading to a uniformly accelerated movement, where the acceleration $\Gamma=2 \gamma / \rho R_{o} a_{o} \simeq 8 \pi \gamma l_{c} / \rho \Omega$ is the ratio of a force to the ring mass $\rho \Omega$. The force acting on these flat rings due to gravity is unusually 


\section{Inertial collapse of liquid rings}

the product of the surface tension $\gamma$ by the capillary length, with a prefactor of $8 \pi$ and a variation as $\gamma^{3 / 2}$. A constant acceleration provides the originality of our system since it is not observed in other situations presenting a similar force balance, such as in inertial capillary rise, break-up of soap films, inertial dewetting or the latter example of the liquid ribbon (figure 7). In all these cases, the liquid in the inertia-capillary regime propagates at constant velocity (de Gennes, Brochard-Wyart \& Quéré 2005). The reason for this difference relies in two key points: (i) We deal with a system where the whole liquid is uniformly put into motion (there are no stagnation points in the flow), unlike the other examples where the volume of liquid in motion increases with time. (ii) The force acting on the liquid ring is constant during most of the closure. With a constant mass and a constant force, the logical outcome is a constant acceleration.

This work raises questions for further investigations concerning the transition between rings and $\mathrm{C}$-shaped puddles, in the limit where the ring is close to a torus. One could also try to do the reverse experiment, starting from a puddle and switching on an annular magnetic field to study the formation of a ring (or a C-shape, depending on radius of the magnetic annulus). Finally, deeper analysis of the flow and the effect of vertical pressure gradient is needed to account for the scaling law observed at the very end of the closure.

\section{Supplementary movie}

Supplementary movie are available at http://dx.doi.org/10.1017/jfm.2013.15.

\section{References}

Aussillous, P. \& Quéré, D. 2004 Shapes of rolling liquid drops. J. Fluid Mech. 512, $133-151$.

Bartolo, D., Josserand, C. \& Bonn, D. 2006 Singular jets and bubbles in drop impact. Phys. Rev. Lett. 96, 124501.

BEMBEnEK, S. D. 2006 Calculations of the surface tension of oxygen using molecular-dynamics simulations. J. Chem. Phys. 124, 014709.

Bergmann, R., VAn der Meer, D., Gekle, S., VAn der Bos, A. \& Lohse, D. 2009 Controlled impact of a disk on a water surface: cavity dynamics. J. Fluid Mech. 633, $381-409$.

Brown, R. A. \& Scriven, L. E. 1980 The shape and stability of rotating liquid drops. Proc. $R$. Soc. Lond. A 371, 331-357.

CHEN, Y.-J. \& STEEN, P. H. 1997 Dynamics of inviscid capillary breakup: collapse and pinchoff of a film bridge. J. Fluid Mech. 341, 245-267.

DAY, R. F., Hinch, E. J. \& LiSTER, J. R. 1998 Self-similar capillary pinchoff of an inviscid fluid. Phys. Rev. Lett. 80, 704-707.

Duclaux, V., Caillé, F., Duez, C., Ybert, C., Bocquet, L. \& Clanet, C. 2007 Dynamics of transient cavities. J. Fluid Mech. 591, 1-19.

EgGers, J. 2011 The subtle dynamics of liquid sheets. J. Fluid Mech. 672, 1-4.

GeKle, S., VAn Der Meer, D., Snoeijer, J. H. \& Lohse, D. 2009 Approach to universality in axisymmetric bubble pinch-off. Phys. Rev. E 80, 036305.

De Gennes, P.-G., Brochard-Wyart, F. \& Quéré, D. 2005 Gouttes, Bulles, Perles et Ondes. Belin, collection Échelles.

Hilgenfeldt, S., Brenner, M. P., Grossmann, S. \& Lohse, D. 1998 Analysis of Rayleigh-Plesset dynamics for sonoluminescing bubbles. J. Fluid Mech. 365, 171-204.

Kojima, M., Hinch, E. J. \& ACRivos, A. 1984 The formation and expansion of a toroidal drop moving in a viscous fluid. Phys. Fluids 27, 19-32. 


\section{B. Darbois Texier, K. Piroird, D. Quéré and C. Clanet}

Leppinen, D. \& Lister, J. R. 2003 Capillary pinch-off in inviscid fluids. Phys. Fluids 15, $568-578$.

LIDE, D. R. 2010 CRC Handbook of Chemistry and Physics, 90th edn. CRC Press/Taylor and Francis.

McGraw, J. D., Li, J., Tran, D. L., Shi, A. C. \& Dalnoki-Veress, K. 2010 Plateau-Rayleigh instability in a torus: formation and breakup of a polymer ring. Soft Matt. 6, 1258-1262.

Nguyen, T. D., Fuentes-Cabrera, M., Fowlkes, J. D., Diez, J. A., GonzÁlez, A. G., Kondic, L. \& RACK, P. D. 2012 Competition between collapse and breakup in nanometre-sized thin rings using molecular dynamics and continuum modelling. Langmuir $\mathbf{2 8 ,}$ $13960-13967$.

PAiram, E. \& Fernandez-Nieves, A. 2009 Generation and stability of toroidal droplets in a viscous liquid. Phys. Rev. Lett. 102, 234501.

Piroird, K., Clanet, C. \& Quéré, D. $2012 a$ Magnetic control of Leidenfrost drops. Phys. Rev. E 85, 056311 .

Piroird, K, Darbois Texier, B., Clanet, C. \& Quéré, D. 2012b Shaping and capturing drops with a magnet. Phys. Fluids (under consideration for publication).

Plateau, J. A. F. 1863 Experimental and theoretical researches on the figures of equilibrium of a liquid mass withdrawn from the action of gravity. In Annual Report of the Board of Regents of the Smithsionian Institution, pp. 270-285.

Plesset, M. S. \& Prosperetti, A. 1977 Bubble dynamics and cavitation. Annu. Rev. Fluid Mech. 9, $145-185$.

Sostarecz, M. C. \& Belmonte, A. 2003 Motion and shape of a viscoelastic drop falling through a viscous fluid. J. Fluid Mech. 497, 235-252.

Worthington, M. A. 1879 On the spontaneous segmentation of a liquid annulus. Proc. R. Soc. Lond. 30, 49-60.

YAO, Z. \& BowICK, M. J. 2011 The shrinking instability of toroidal liquid droplets in the Stokes flow regime. Eur. Phys. J. E 34, 1-6. 